\title{
The plasma ammonia response to cycle exercise in COPD
}

\author{
L.D. Calvert*, S.J. Singh*, P.L. Greenhaff* ${ }^{*}$ M.D. Morgan* and M.C. Steiner*
}

\begin{abstract}
The plasma ammonia response to exercise in chronic obstructive pulmonary disease (COPD) was examined and the relationship between plasma ammonia concentration and muscle adenine nucleotide metabolism was explored.
\end{abstract}

In total, 25 stable COPD patients and 13 similar-aged controls underwent incremental and constant-work rate cycle exercise tests. Arterialised venous blood was sampled at rest, at 1-min intervals during exercise and $\leqslant \mathbf{5} \mathrm{min}$ after exercise for ammonia and lactate concentration.

Peak incremental work rate was significantly less in COPD subjects (67 $\pm 21 \mathrm{~W})$ than similar-aged controls (156 $\pm 46 \mathrm{~W})$. In COPD and control subjects, plasma ammonia concentration increased during incremental exercise until 2 min post-exercise and then declined by 5 min post-exercise. However, two distinct patterns were seen in COPD subjects. In one group $(n=16)$, ammonia increased $\left(42.8 \pm 3.3 \mu \mathrm{mol} \cdot \mathrm{L}^{-1}\right)$ by a similar magnitude as the controls $\left(55.5 \pm 7.0 \mu \mathrm{mol} \cdot \mathrm{L}^{-1}\right)$. In the second COPD group $(n=9)$, no ammonia increase was observed despite a similar lactate increase. Ammonia change with incremental and constant-work rate exercise strongly correlated in COPD subjects. Plasma ammonia increase correlated with muscle inosine-5'-monophosphate formation after constant-work rate exercise.

Plasma ammonia concentration increases during incremental and constant-work rate cycle exercise in chronic obstructive pulmonary disease subjects at lower absolute work rates compared with similar-aged controls. The plasma ammonia response may provide useful information about adenine nucleotide metabolism and, therefore, muscle fatigue during exercise in patients with chronic obstructive pulmonary disease.

KEYWORDS: Adenine nucleotides, chronic obstructive pulmonary disease, metabolic response, skeletal muscle dysfunction

\begin{abstract}
A bnormal peripheral muscle function has been identified as an important contributor to exercise intolerance in patients with chronic obstructive pulmonary disease (COPD). This is independent of disease severity and linked to disability, poor quality of life and survival [1]. Quadriceps muscle samples, taken from COPD patients at rest, show reductions in oxidative enzyme activity and the proportion of type I fibres compared with age-matched healthy controls [2-4]. An accelerated rise in blood lactate during exercise in COPD has been reported compared with control subjects and is associated with a reduction in quadriceps muscle mitochondrial enzyme activity [5]. This implies that resynthesis of adenosine triphosphate (ATP) during muscle contraction from oxidative sources is impaired in COPD and results in an increase in nonoxidative metabolism and, presumably, fatigue
\end{abstract}

For editorial comments see page 693. during exercise. Importantly, this impairment in skeletal muscle energy metabolism may be a remediable feature of an otherwise largely irreversible pulmonary disease.

It has previously been shown in COPD that ATP degradation and inosine-5'-monophosphate (IMP) accumulation in skeletal muscle during exercise occurs despite the significantly lower absolute work rates (WRs) that these individuals can achieve [6]. This suggests that metabolic stress occurs in patients with COPD at these low absolute WRs, which may be relevant to their daily living activities. However, there was significant inter-individual variability in the magnitude of the metabolic response, and further understanding of the characteristics and mechanisms underlying the skeletal muscle metabolic response to exercise is required. Measurement of metabolic events during exercise may be an important investigational tool, but obtaining muscle biopsies during exercise is technically
AFFILIATIONS

*Dept of Respiratory Medicine, Institute for Lung Health, Glenfield Hospital, Leicester, and "Dept of Biomedical Sciences, University of Nottingham, Nottingham, UK.

CORRESPONDENCE

L.D. Calvert

Dept of Respiratory Medicine Glenfield Hospital

Groby Road

Leicester

LE3 9QP

UK

Fax: 441162367768

E-mail: Iori.calvert@uhl-tr.nhs.uk

Received:

December 152006

Accepted after revision:

November 242007

SUPPORT STATEMENT

The study received financial support from the University Hospitals of Leicester NHS Trust.

STATEMENT OF INTEREST None declared. 
difficult in this frail, elderly population and, therefore, not practical for larger clinical trials. Alternative methods for studying the metabolic response are needed.

During intense exercise, ATP degradation occurs when oxidative and nonoxidative ATP re-synthesis fails to meet ATP demand. This is associated with accumulation of IMP as a result of irreversible deamination of adenosine monophosphate (AMP) in exercising skeletal muscle. This process has been described in the literature as metabolic stress $[7,8]$. During this reaction, ammonia is produced in stochiometry with IMP and released into the bloodstream. In young healthy adults, blood ammonia concentration has been shown to increase during incremental exercise only when high intensities are reached $[9,10]$. This has been implicated in the development of fatigue and physical exhaustion [11]. Although plasma ammonia has been shown to closely reflect muscle adenine nucleotide metabolism in healthy subjects [11], the ammonia response to exercise in subjects with COPD has not been reported.

It was hypothesised that in COPD subjects, changes in ammonia concentration during exercise would reflect adenine nucleotide metabolism within skeletal muscle and provide a useful marker of skeletal muscle metabolism, which is less invasive than obtaining a muscle biopsy. The present study examined the plasma ammonia response to both incremental and constant-WR cycle exercise in COPD, and explored the relationship between plasma ammonia concentration and skeletal muscle adenine nucleotide metabolism.

\section{METHODS}

In total, 25 stable patients with COPD (aged 50-85 yrs) who met Global Initiative for Chronic Lung Disease criteria [12] were recruited from outpatient clinics. Patients were excluded if they: were taking maintenance oral corticosteroids; were unable to perform exercise tests; demonstrated exercise desaturation $(<85 \%)$; had significant cardiac dysfunction; had an exacerbation of COPD within the previous 6 weeks or pulmonary rehabilitation within the last year. Similar-aged healthy controls $(n=13)$ were recruited by local advertisement and screened for abnormal lung function and significant cardiac or respiratory disease. Full approval was obtained from the Leicestershire Research Ethics Committee (Leicester, UK) and all participants provided informed written consent.

\section{Study design}

Participants attended an initial visit to collect baseline data and to familiarise themselves with the exercise test. On a subsequent visit $\leqslant 72 \mathrm{~h}$ later, subjects performed a maximal (symptom-limited) incremental exercise test on an electrically braked cycle ergometer. Subjects then performed a constantWR exercise challenge 1 week later.

\section{Baseline measurements}

Spirometry was performed on three occasions in the seated position (Model R; Vitalograph, Buckingham, UK) according to standards set out by the European Respiratory Society [13]. Body mass index was calculated from height (measured using a wall-mounted stadiometer (SECA, Birmingham, UK) to the nearest $0.1 \mathrm{~cm}$ ) and weight (measured in light clothing to the nearest $0.1 \mathrm{~kg}(\mathrm{SECA})$ ). Fat-free mass (measured in $\mathrm{kg}$ ) was estimated using bioelectrical impedance when subjects were in the semi-supine position, and calculated using disease-specific equations [14]. Isometric quadriceps force was evaluated using the Cybex II Norm dynamometer (CYBEX NORM ${ }^{\mathrm{TM}}$ Testing and Rehabilitation System; CYBEX International Inc., Medway, MA, USA), with subjects seated at a $70^{\circ}$ knee flexion. Physical activity was assessed using a physical activity questionnaire adapted for the elderly [15] and validated in subjects with COPD [16]. The questionnaire consisted of scores for household activities, sport activities and leisure activities, resulting in an overall activity score.

\section{Exercise challenge}

In the incremental test, $\mathrm{WR}$ was increased by $10 \mathrm{~W}$ every minute (COPD) or $20 \mathrm{~W}$ every minute (healthy) using a ramp protocol to determine peak exercise work capacity (Ergometric Er900; Ergoline GmbH, Bitz, Germany). Participants cycled at a cadence of 40-45 rpm and were encouraged to continue cycling at the required rate for as long as possible. Ventilation and gas exchange measurements were performed throughout the test using a breath-by-breath computerised system (Zan-680 ErgoTest; Zan Messgeraete $\mathrm{GmbH}$, Oberthulba, Germany). Peak ventilation was expressed as a percentage of maximum voluntary ventilation (MVV) and the ventilation of patients with COPD was deemed limited if peak ventilation exceeded $90 \%$ MVV [17]. In the constant-WR test, work increased over $1 \mathrm{~min}$ then, subjects cycled at a constant WR until symptom-limited. The intensity for this test was set at $80 \%$ of the peak work achieved during the incremental test.

\section{Blood/biopsy analysis}

A 12-g retrograde cannula was inserted into a superficial lower forearm vein $30 \mathrm{~min}$ prior to exercise and placed inside a hand warmer then warmed to $50-55^{\circ} \mathrm{C}$. The hand warmer enables arterialised venous blood to be collected, which is representative of arterial blood and is therefore not contaminated by ammonia generated by the hand and forearm muscles. This method has been previously validated and used for plasma ammonia measurements in healthy subjects $[18,19]$. Arterialised venous-blood samples were taken at rest (subjects rested for $30 \mathrm{~min}$ ), every minute during exercise to peak exercise, and at 2 and $5 \mathrm{~min}$ after exercise. All samples were immediately placed on ice. Blood for ammonia analysis was centrifuged immediately following the exercise test, plasma was stored at $-196^{\circ} \mathrm{C}$ in liquid nitrogen, and analysed in duplicate usually immediately, but always within $24 \mathrm{~h}$, using a validated enzyme assay technique (Sigma-Aldrich Co. Ltd, Gillingham, UK). The coefficient of variation for ammonia determined from standards was $5 \%$. Whole blood lactate concentrations were analysed immediately following exercise using a bench-top analyser (YSI 1500 sport 1lactate analyser; YSI Inc., San Diego, CA, USA). The coefficient of variation for lactate determined from standards was $2 \%$.

Muscle biopsies of the vastus lateralis were obtained, using the technique described by BERGSTROM [20], at rest (subjects rested for $30 \mathrm{~min}$ ) and immediately post-exercise (within $10 \mathrm{~s}$ of peak exercise while seated on the bike) following the constant-WR challenge. This meant that muscle samples were taken following an exercise challenge of the same relative intensity for all subjects. Muscle samples were frozen and stored immediately in liquid nitrogen. Following subsequent freeze 
drying, powdering and extraction, samples were analysed for phosphocreatine (PCr) and creatine concentrations using the spectrophotometric method of HARRIS et al. [21]. Adenine nucleotides (ATP, adenosine diphosphate (ADP) and AMP) and their breakdown derivatives (IMP, inosine and xanthine) were measured using high-pressure liquid chromatography [22]. Total creatine concentration was calculated as the sum of $\mathrm{PCr}$ and creatine. All measurements were corrected for nonmuscle constituents using total creatine [23].

\section{Data analysis}

Between-group comparisons were made using an unpaired t-test or the Mann-Whitney U-test when not normally distributed. Within-group comparisons were performed using paired t-tests. Correlations between parameters were calculated with Pearson's correlation tests. Significance was assumed at $\mathrm{p}<0.05$.

\section{RESULTS}

\section{Patient characteristics}

A total of 25 patients with COPD and 13 similar-aged controls were included. One COPD patient dropped out after familiarisation and was not included in analyses. Missing data in the incremental test were due to equipment failure (two control, two COPD) and insufficient blood for accurate analysis (one control). Missing data in the constant-WR test were due to equipment failure (one control, three COPD), intolerance of procedure (two COPD) and insufficient biopsy material (three control, seven COPD). Baseline characteristics for COPD and control subjects are shown in table 1 and presented as mean when normally distributed and median when not normally distributed. Demographically, the groups were well matched apart from forced expiratory volume in one second and physical activity score, which were expected.

\section{Incremental exercise}

Data from the incremental exercise test is shown in table 1. Peak WR (mean $\pm \mathrm{SD}$ ) was significantly lower in subjects with COPD $(67.2 \pm 20.5 \mathrm{~W})$ than similar-aged controls $(156.2 \pm 45.7 \mathrm{~W}$; $p<0.001)$. Peak ventilation was significantly increased in subjects with COPD compared with similar-aged controls, and five COPD subjects suffered limited ventilation.

Tables 2 and 3 show mean \pm SD plasma ammonia and blood lactate responses to incremental cycle exercise. Resting plasma ammonia concentrations were similar for COPD and similaraged subjects and within published ranges [7, 9, 24]. Plasma ammonia concentration increased during exercise in subjects with COPD $(p<0.001)$ and similar-aged subjects $(p<0.001)$ and continued to increase at $2 \mathrm{~min}$ after exercise, before declining towards baseline at $5 \mathrm{~min}$ after exercise.

In COPD subjects two distinct patterns of response appeared when plasma ammonia increased, with incremental exercise plotted against peak oxygen uptake (fig. 1a). In one group of COPD subjects plasma ammonia increased significantly with exercise and change in ammonia concentration correlated with peak oxygen uptake (Pearson correlation $r=0.56, p=0.03$ ). The ammonia increase in control subjects also correlated with oxygen consumption $(\mathrm{r}=0.56, \mathrm{p}=0.07)$. In a second group of subjects with COPD, ammonia did not increase with exercise despite subjects achieving similar peak oxygen uptake $\left(17.1 \pm 4.2 \mathrm{~mL} \cdot \mathrm{kg}^{-1} \cdot \mathrm{min}^{-1}\right.$ versus $17.3 \pm 3.0 \mathrm{~mL} \cdot \mathrm{kg}^{-1} \cdot \mathrm{min}^{-1} \quad(\mathrm{p}=0.88)$ in the group with ammonia increase) and peak WR $(63 \pm 19 \mathrm{~W}$ versus $69 \pm 21 \mathrm{~W}$ $(\mathrm{p}=0.45)$ in the group with ammonia increase). However, no such differential response was seen with blood lactate (fig. 1b), and the change in blood lactate during exercise was not significantly different between the two groups of COPD subjects $(p=0.30$; table 3). The magnitude of blood lactate accumulation was correlated with peak oxygen uptake as expected $(r=0.55$, $p=0.007)$. The differential ammonia response in COPD subjects could not be predicted from demographic variables, medication or exercise parameters, including limitations to exercise and ventilatory limitation, which were not significantly different between the two groups.

Figure 2 shows the pattern of change in plasma ammonia from resting concentration to peak WR during incremental exercise for all control subjects, COPD subjects with an ammonia rise and COPD subjects with no ammonia rise. In age-matched controls, plasma ammonia concentration remained near resting concentration at low WRs. At higher intensity exercise, ammonia concentration increased with increasing WR. In COPD subjects with an ammonia response, plasma ammonia concentration increased from the onset of exercise and continued to rise with increasing WR.

The increase in plasma ammonia concentration during exercise $\left(\right.$ mean \pm SE $\left.42.8 \pm 3.3 \mu \mathrm{mol} \cdot \mathrm{L}^{-1}\right)$ in $15 \mathrm{COPD}$ subjects with a response was not significantly different to similar-aged controls (mean \pm SE $55.5 \pm 7.0 \mu \mathrm{mol} \cdot \mathrm{L}^{-1} ; \mathrm{p}=0.12$ ), despite the significantly lower absolute peak WR achieved (tables 2 and 3). However, the increase in blood lactate in these COPD subjects was significantly lower than control subjects $(\mathrm{p}<0.001)$. In the COPD group $(\mathrm{n}=9)$ with no measurable ammonia increase, the change in plasma ammonia concentration was within repeatability of the measurement. Unlike the present findings in similar-aged subjects where there was a linear relationship between peak ammonia and lactate concentrations $(r=0.61, p=0.046)$, there was no relationship between plasma ammonia and blood lactate concentration in all subjects with COPD $(r=0.02, p=0.938)$.

\section{Constant-WR exercise}

Subjects with or without a plasma ammonia increase in the incremental exercise challenge had a consistent response in the constant-WR exercise challenge. Change in plasma ammonia concentration during incremental exercise strongly correlated with plasma ammonia change during constant-WR exercise in all subjects with COPD $(r=0.88, p<0.001$; fig. 3$)$.

\section{Biopsy data}

ATP degradation and IMP accumulation occurred in skeletal muscle during constant WR exercise in all COPD subjects $(n=14$; mean \pm SD change $-3.11 \pm 1.41 \mathrm{mmol} \cdot \mathrm{kg}^{-1}(\mathrm{p}=0.046)$ dry weight and $0.58 \pm 0.23 \mathrm{mmol} \cdot \mathrm{kg}^{-1}(\mathrm{p}=0.029)$ dry weight, respectively) and similar-aged controls $\left(n=9 ;-4.44 \pm 1.42 \mathrm{mmol} \cdot \mathrm{kg}^{-1}(\mathrm{p}=0.019)\right.$ dry weight and $2.86 \pm 0.81 \mathrm{mmol} \cdot \mathrm{kg}^{-1} \quad(\mathrm{p}=0.01)$ dry weight, respectively). The absolute WRs were significantly different between COPD subjects and similar-aged controls $(52 \pm 17 \mathrm{~W}$ and $128 \pm 38 \mathrm{~W}$, respectively; $\mathrm{p}<0.001)$. $\mathrm{PCr}$ and $\mathrm{PCr} /$ creatine ratio fell significantly and to a similar extent in COPD subjects and controls. PCr concentrations pre- and post-exercise were $72.1 \pm 12.8 \mathrm{mmol} \cdot \mathrm{kg}^{-1}$ and $53.4 \pm 18.3 \mathrm{mmol} \cdot \mathrm{kg}^{-1}$ dry weight, respectively, in COPD subjects and $70.9 \pm 7.4 \mathrm{mmol} \cdot \mathrm{kg}^{-1}$ and 


\begin{tabular}{|c|c|c|c|}
\hline \multirow[t]{2}{*}{ TABLE 1} & \multicolumn{3}{|c|}{$\begin{array}{l}\text { Baseline characteristics and exercise data from } \\
\text { incremental cycle test for similar-aged control } \\
\text { and chronic obstructive pulmonary disease } \\
\text { (COPD) subjects }\end{array}$} \\
\hline & & Controls & COPD \\
\hline \multicolumn{2}{|l|}{ Subjects $n$} & 13 & 25 \\
\hline \multicolumn{2}{|l|}{ Age yrs } & $68 \pm 7$ & $69 \pm 7$ \\
\hline \multicolumn{2}{|l|}{ Sex $M / F$} & $10 / 3$ & $20 / 5$ \\
\hline \multicolumn{2}{|c|}{ FEV $1 \%$ pred } & $101 \pm 16$ & $47 \pm 12^{\star \star \star}$ \\
\hline \multicolumn{2}{|l|}{ FEV 1} & $2.88 \pm 0.71$ & $1.21 \pm 0.29 * \star \star$ \\
\hline \multicolumn{2}{|c|}{ Isometric quadriceps strength $\mathrm{Nm}$} & $155 \pm 52$ & $130 \pm 47$ \\
\hline \multicolumn{2}{|l|}{ FFMI $\mathbf{~ k g} \cdot \mathbf{m}^{-2}$} & $19 \pm 3$ & $18 \pm 2$ \\
\hline \multicolumn{2}{|l|}{$\mathrm{BMI} \mathbf{k g} \cdot \mathrm{m}^{-2}$} & $26 \pm 4$ & $27 \pm 4$ \\
\hline \multicolumn{2}{|c|}{ Physical activity score } & $15.7(10.3)$ & $5.7(5.8) * \star \star$ \\
\hline \multicolumn{2}{|c|}{ fc \% pred } & $93 \pm 7$ & $73 \pm 10^{\star \star \star}$ \\
\hline \multicolumn{2}{|l|}{ Peak WR W } & $156 \pm 46$ & $67 \pm 21^{* \star *}$ \\
\hline \multicolumn{2}{|c|}{ Peak $V^{\prime} \mathrm{O}_{2} \mathrm{~mL} \cdot \mathbf{k g}^{-1} \cdot \mathrm{min}^{-1}$} & $28.50 \pm 8.25$ & $17.21 \pm 3.41^{* \star *}$ \\
\hline \multicolumn{2}{|c|}{ Peak V'E L. $\mathrm{min}^{-1}$} & $74 \pm 21$ & $37 \pm 10^{\star \star \star}$ \\
\hline \multicolumn{2}{|c|}{ Peak V'E \% MVV } & $66 \pm 16$ & $79 \pm 20^{\star}$ \\
\hline \multicolumn{2}{|l|}{ Peak RQ } & $1.14 \pm 0.15$ & $0.96 \pm 0.07$ \\
\hline \multicolumn{2}{|l|}{ Peak PE } & $16(6)$ & $16(9)$ \\
\hline
\end{tabular}

Data are expressed as mean \pm SD or median (interquartile), unless otherwise stated. M: males; F: females; FEV1: forced expiratory volume in one second; \% pred: \% predicted; FFMI: fat-free mass index; BMI: body mass index; fC: cardiac frequency; WR: work rate; $V^{\prime} \mathrm{O}_{2}$ : peak oxygen uptake; $V^{\prime} E$ : minute ventilation; MVV: maximum voluntary ventilation, calculated as $\mathrm{FEV}_{1} \times 40$; $R Q$ : respiratory quotient; PE: perceived exertion at peak exercise. ${ }^{*}: p<0.05$; ***: $\mathrm{p}<0.001$ compared with controls.

$40.0 \pm 10.3 \mathrm{mmol} \cdot \mathrm{kg}^{-1}$ dry weight, respectively, in controls. $\mathrm{PCr} /$ creatine ratios pre- and post-exercise were $1.36 \pm 0.33$ and $0.80 \pm 0.36$, respectively, in COPD subjects and $1.43 \pm 0.23$ and $0.53 \pm 0.21$, respectively, in controls. Insufficient tissue was available for analysis of other purine nucleotide derivatives. No statistically significant differences were seen in the exerciseinduced change in muscle metabolites between the two COPD subgroups (with and without an ammonia response); although, due to missing biopsy data, numbers were small.

Correlation existed between muscle IMP accumulation and plasma ammonia increase in subjects with COPD $(r=0.61$, $\mathrm{p}=0.029)$ and similar-aged controls $(\mathrm{r}=0.66, \mathrm{p}=0.055)$. Figure 4 demonstrates these correlations. No correlation was found between plasma ammonia increase and muscle ATP degradation or between muscle IMP accumulation and ATP degradation in either COPD or control subjects.

\section{DISCUSSION}

The present study is the first to describe the plasma ammonia response to cycle exercise in COPD. Overall, a significant exercise-induced increase in plasma ammonia concentration was found, which began early in exercise and peaked 2 min after exercise. Similar-aged controls displayed a curvilinear ammonia response to incremental exercise, which was similar to findings documented in the literature for young healthy subjects $[9,10]$. However, there was a differential ammonia response to exercise in the COPD cohort. In one subgroup, the increase in plasma ammonia from rest to end exercise was similar to controls despite significantly lower peak WRs. The other subgroup of COPD subjects did not demonstrate an increase in plasma ammonia concentration despite having an increase in blood lactate concentration.

Failure of energy delivery by oxidative and anaerobic ATP re-synthesis to meet the demands of muscle force generation results in an increase in ADP and AMP, and activation of AMP deaminase. The irreversible deamination of AMP leads to accumulation of IMP in exercising muscles and release of ammonia into the bloodstream. In the short term, by preventing excessive accumulation of ADP and AMP, this increases the phosphorylation potential of the adenine nucleotide pool, allowing the adenylate kinase reaction and contraction to continue. Such a situation is not sustainable due to the resulting accumulation of ADP and decline in ATP availability. Substantial metabolic stress is said to have occurred under these conditions $[6,7]$ and is associated with fatigue in healthy subjects $[25,26]$. It has previously been shown that ATP loss and IMP accumulation in muscle occurs at significantly lower absolute WRs in subjects with COPD than healthy subjects [6]. Data from the present study support these findings and demonstrate a rise in ammonia with exercise at lower absolute WRs compared with similar-aged control subjects. The data support the authors' previous observations that skeletal muscles in subjects with COPD are working under conditions of metabolic stress at low absolute work intensities, similar to those required for activities of daily living [6]. The increase in

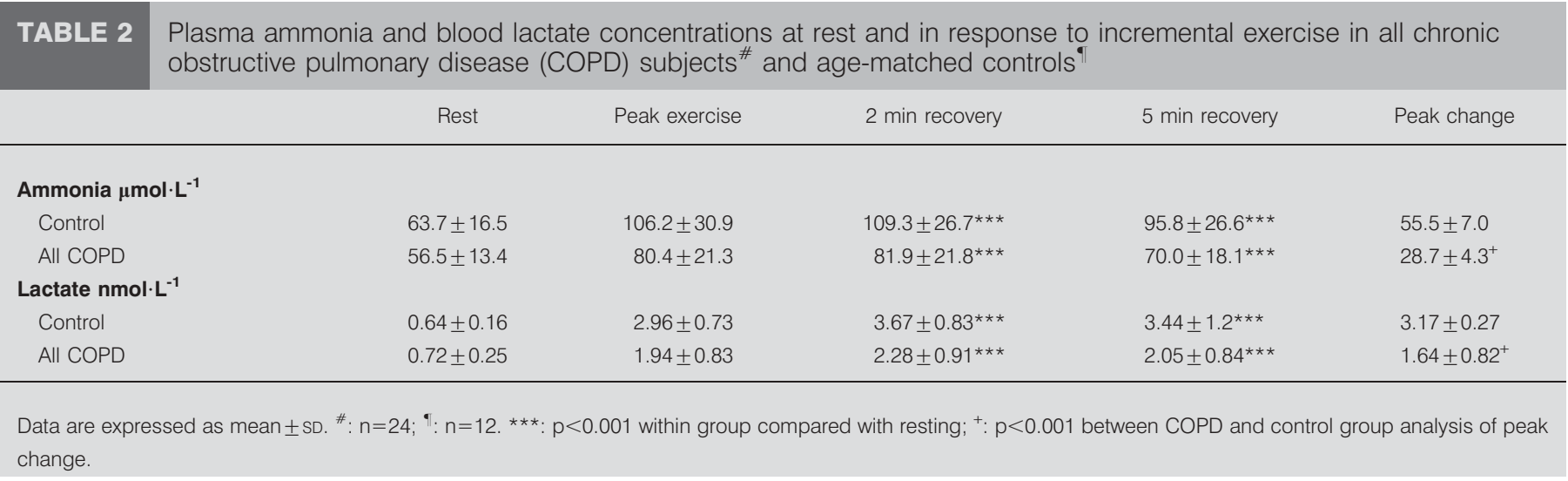




\begin{tabular}{|c|c|c|c|c|c|c|}
\hline TABLE 3 & \multicolumn{6}{|c|}{$\begin{array}{l}\text { Plasma ammonia and blood lactate concentrations at rest and in response to incremental exercise in all chronic } \\
\text { obstructive pulmonary disease (COPD) subjects with (group 1) }{ }^{\#} \text { and without (group 2) an ammonia response during } \\
\text { exercise }\end{array}$} \\
\hline \multicolumn{7}{|c|}{ Ammonia $\mu \mathrm{mol} \cdot \mathrm{L}^{-1}$} \\
\hline COPD group & & $55.9 \pm 12.5$ & $91.3 \pm 18.4^{\star \star \star}$ & $93.0 \pm 19.2^{\star \star \star}$ & $77.1 \pm 18.7^{\star \star \star}$ & $42.8 \pm 3.3$ \\
\hline COPD grour & & $0.70 \pm 0.23$ & $2.00 \pm 0.89 * * \star$ & $2.36 \pm 0.97^{\star \star \star}$ & $2.18 \pm 0.91 * \star \star$ & $1.78 \pm 0.21^{+}$ \\
\hline COPD grour & & $0.77 \pm 0.30$ & $1.84 \pm 0.73^{\star \star}$ & $2.16 \pm 0.88^{\star \star \star}$ & $1.81 \pm 0.68^{\star \star \star}$ & $1.39 \pm 0.30^{+}$ \\
\hline
\end{tabular}

plasma ammonia correlated with muscle IMP accumulation in constant-WR exercise supports the present authors' hypothesis that plasma ammonia may be a useful marker of the nucleotide metabolic response within skeletal muscle.

a)

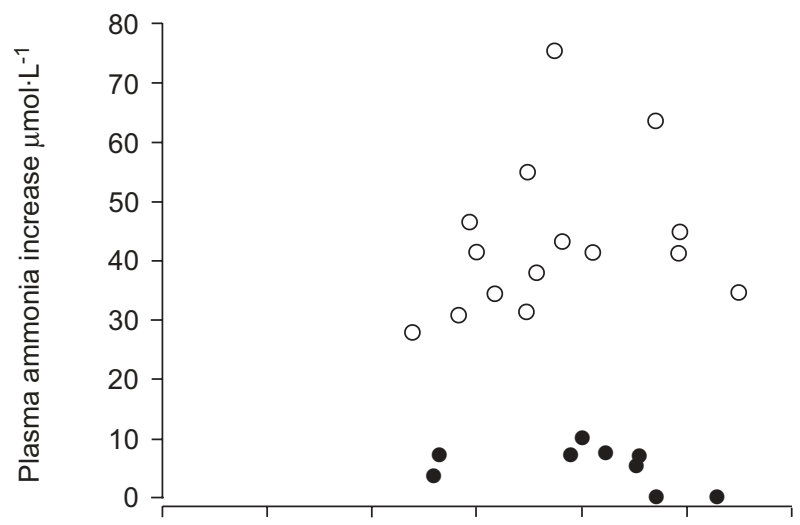

b)

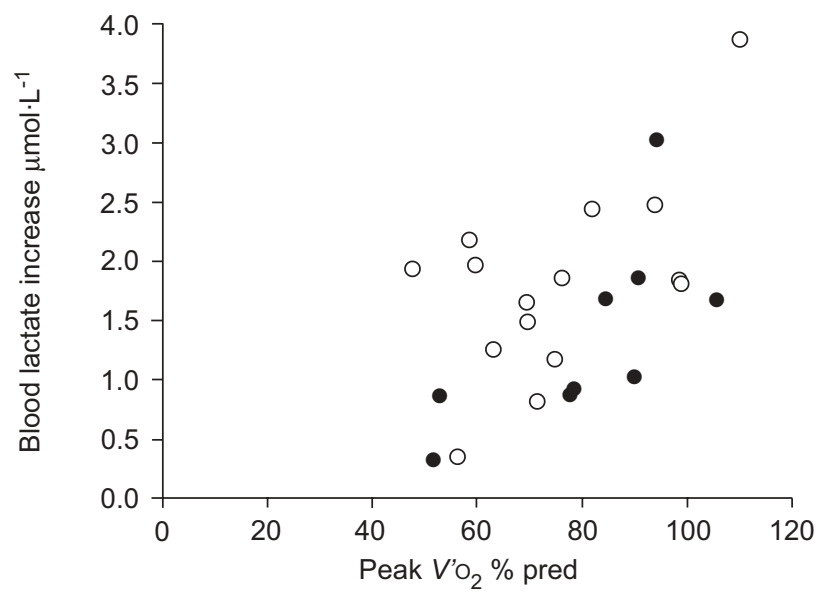

FIGURE 1. Peak oxygen uptake $\left(V^{\prime} \mathrm{O}_{2}\right)$ of a) maximum plasma ammonia change and $b$ ) maximum blood lactate change following maximal incremental cycle exercise in 24 chronic obstructive pulmonary disease subjects. \% pred: \% predicted. $\mathrm{O}$ : subjects with an ammonia response $(n=15)$; : subjects without an ammonia response $(n=9)$.
The main source of plasma ammonia produced in skeletal muscles during intense exercise is from deamination of AMP, which constitutes part of the purine nucleotide cycle (PNC). It has been demonstrated in humans that the activity of the PNC and blood ammonia production is predominantly a reflection of fast twitch (type II) fibre activity during short-term intense exercise [27]. DuDLEY et al. [28] reported an inverse relationship between the proportion of slow twitch (type I) fibres of the vastus lateralis muscle and ammonia increase during intense exercise in healthy subjects. The current findings of an early increase in plasma ammonia in incremental exercise in subjects with COPD suggest that fast twitch fibre recruitment is occurring at low WRs in this population. Atrophy of type I fibres and an increased proportion of type II fibres in skeletal muscle samples taken at rest in patients with COPD is well recognised $[2,3]$.

A reduction in oxidative enzyme concentrations in muscles of subjects with COPD has been demonstrated at rest $[4,5]$. This implies either reduced oxidative ATP metabolism and/or increased reliance on anaerobic ATP re-synthesis during

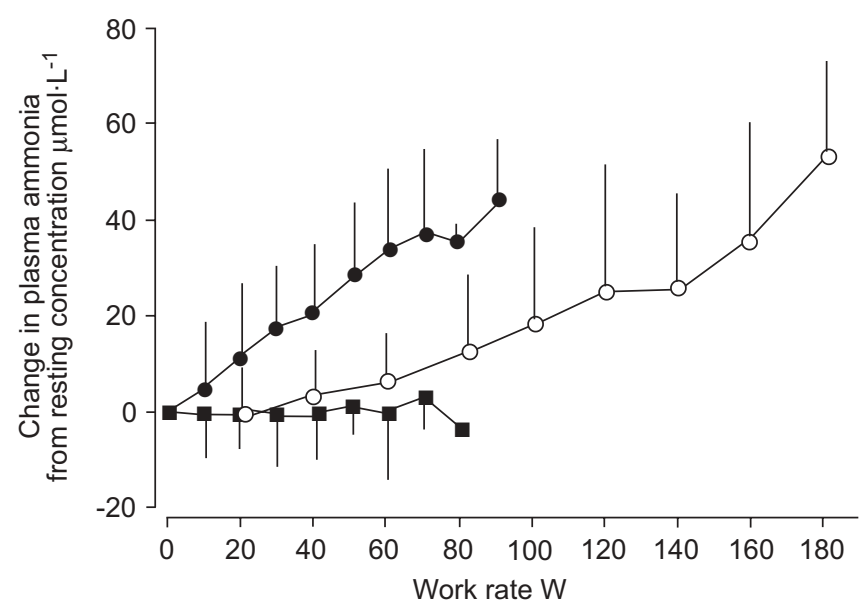

FIGURE 2. Mean $\pm S D$ change in plasma ammonia concentration from resting concentration during incremental exercise in chronic obstructive pulmonary disease subjects with $(\bullet: n=15)$ and without ammonia increase $(\boldsymbol{\square}: n=9)$ and similar-aged controls $(0: n=12)$. 


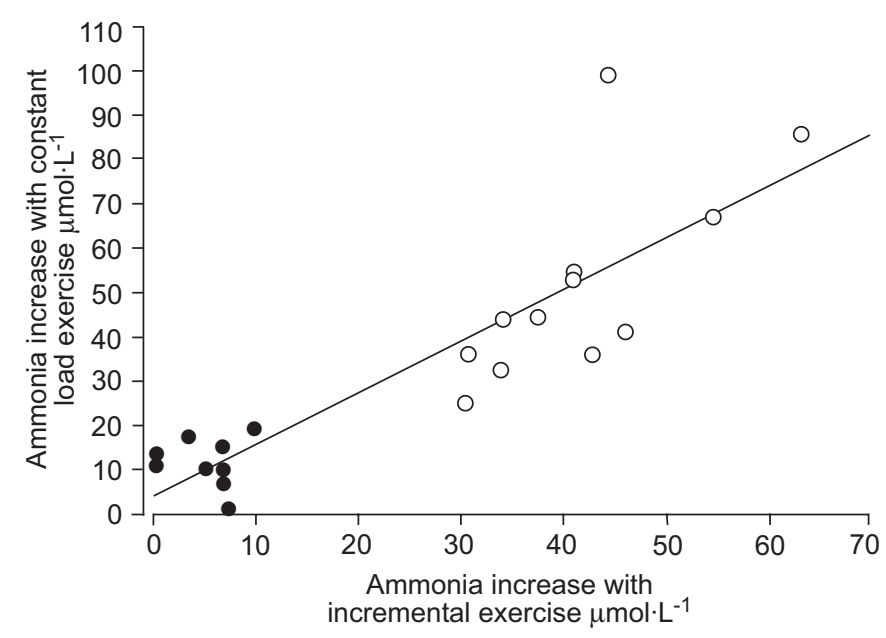

FIGURE 3. A scatter graph to show correlation between maximum plasma ammonia change during maximal incremental and sub-maximal constant-work rate cycle exercise in 21 chronic obstructive pulmonary disease (COPD) subjects. $\bigcirc$ : COPD subjects with ammonia response $(n=12) ; 0$ : COPD subjects without ammonia response $(n=9)$. $R^{2}=0.766$.

exercise, or a preferential atrophy of oxidative muscle fibres. Increased reliance on glycolytic metabolism has recently been associated with contractile fatigue following cycle exercise [29]. Further support for this is provided by MALTAIS et al. [5], who showed an early and accelerated blood lactate accumulation during incremental exercise in severe COPD. Although blood lactate concentration can be used as a marker of metabolic response to exercise, plasma ammonia may more closely reflect changes in adenine nucleotide metabolism occurring under conditions of metabolic stress.

It was unclear from the present data why some subjects with COPD failed to display an ammonia response during exercise. The current authors were unable to identify differences in demographics, disease severity or the pattern of exercise response between these patients and those who did show a rise

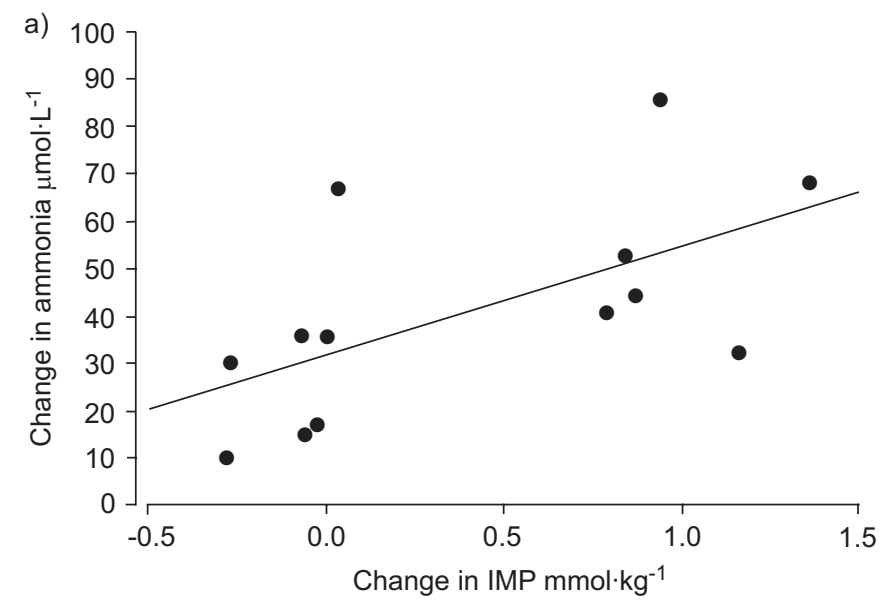

in ammonia. Missing biopsy samples rendered interpretation of exercise-induced metabolite changes difficult between the two COPD subgroups due to the small sample size. However, an increase in blood lactate does not necessarily have to be matched by an increase in plasma ammonia. One possibility is that, in patients without a rise in ammonia, the ATP demands of contraction were being met and fatigue was attributable to another factor not associated with the failure of energy delivery. An alternative explanation is that these patients may have differed in muscle fibre composition, such that considerably less ammonia was generated. In this respect, human slow twitch muscle fibres are known to have considerably less deamination of AMP to IMP and, therefore, less ammonia generation. Previous literature in COPD has suggested a shift in fibre composition towards a greater proportion of type II fibres [2, 3], but this phenomenon may vary considerably across the COPD population and it is possible that patients not showing a rise in ammonia were those with better preservation of type I (slow twitch) fibres. This was a post hoc analysis and, as such, does need to be confirmed in future studies, together with measurements of muscle fibre composition, blood flow and oxidative enzyme concentrations to explain these observations.

A number of limitations to the current study are acknowledged, particularly in interpreting the muscle biopsy data. As has been found in previous studies, tissue from biopsies taken immediately post-exercise was small and, in some cases, inadequate for complete analysis. This highlights the technical difficulties and limitations in using muscle biopsies to investigate the metabolic response to exercise in COPD. Biopsies were taken following constant-WR exercise, which has been shown in previous work [6] to induce skeletal muscle metabolic stress. As the WR for the constant-WR test was determined by performance during the incremental test, the metabolic response to exercise measured in the muscles will have been influenced by the limit to maximal performance. This highlights some of the problems with standardising submaximal exercise tests for studies. However, an intensity of $80 \%$ work achieved in the maximal incremental test was felt to b)

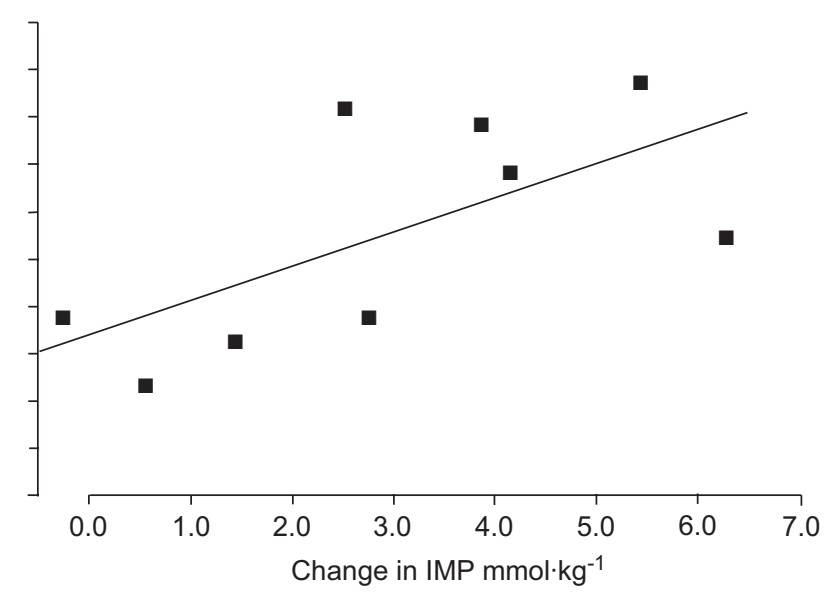

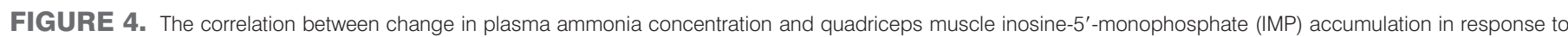

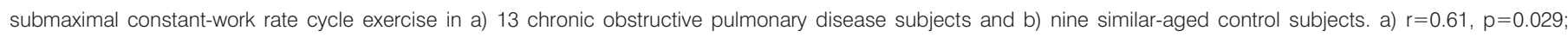
b) $r=0.66, p=0.055$. 
be appropriate for several reasons. It reflects the intensity at which patients are asked to perform endurance training in rehabilitation and, therefore, has some practical relevance. In addition, similar work intensities have been demonstrated to stimulate an ammonia response to submaximal exercise in healthy subjects [27]. Although muscle ammonia was not directly measured in the current study, a strong relationship has been shown to exist between muscle adenine nucleotide loss and plasma ammonia accumulation [30]. Measurements from forearm arterialised blood may be less sensitive than those taken from femoral venous blood as this directly drains the exercising muscles, but the aim of the present study was to evaluate less invasive measurements of ammonia that may be practical as an investigational tool in clinical studies. For this reason femoral venous cannulation was not carried out. Finally, while the control group were not physically well trained and were representative of the healthy elderly population, they were significantly less active than the COPD group. Thus, the present authors are unable to distinguish the effects of loss of fitness due to inactivity from other aspects of the disease on the aetiology of the observations.

COPD is a leading cause of disability worldwide and places an increasing burden on healthcare resources [12]. Peripheral muscle dysfunction and, in particular, impaired energy metabolism may prove an important remediable source of exercise intolerance in this population despite largely irreversible lung impairment. In healthy subjects, training increases ammonia workload threshold [31] and a reduction in blood ammonia concentration appears to delay onset of fatigue and increase the duration of intense exercise $[11,31]$. It is feasible that similar results with training could be achieved in COPD patients who have an observed ammonia increase with exercise. Plasma ammonia may be a marker of metabolic stress in the skeletal muscles and, therefore, could be used as an outcome when assessing the impact of interventions targeting skeletal muscle energy metabolism, such as pulmonary rehabilitation.

In conclusion, the current authors have shown that plasma ammonia concentration increases during incremental and constant-work rate cycle exercise in subjects with chronic obstructive pulmonary disease. Compared with similar-aged controls, similar peak exercise ammonia concentrations are reached despite significantly lower peak work rates. The observed differential ammonia response to cycle exercise appears to be distinct from the lactate response and may provide a useful clinical marker for investigating differences in skeletal muscle energy metabolism during exercise in chronic obstructive pulmonary disease patients.

\section{REFERENCES}

1 American Thoracic Society. Skeletal muscle dysfunction in chronic obstructive pulmonary disease. A statement of the American Thoracic Society and European Respiratory Society. Am J Respir Crit Care Med 1999; 159: S1-S40.

2 Whittom F, Jobin J, Simard PM, et al. Histochemical and morphological characteristics of the vastus lateralis muscle in patients with chronic obstructive pulmonary disease. Med Sci Sports Exerc 1998; 30: 1467-1474.
3 Gosker HR, Engelen MP, van Mameren H, et al. Muscle fiber type IIX atrophy is involved in the loss of fat-free mass in chronic obstructive pulmonary disease. Am J Clin Nutr 2002; 76: 113-119.

4 Maltais F, LeBlanc P, Whittom F, et al. Oxidative enzyme activities of the vastus lateralis muscle and the functional status in patients with COPD. Thorax 2000; 55: 848-853.

5 Maltais F, Simard AA, Simard C, Jobin J, Desgagnes P, LeBlanc P. Oxidative capacity of the skeletal muscle and lactic acid kinetics during exercise in normal subjects and in patients with COPD. Am J Respir Crit Care Med 1996; 153: 288-293.

6 Steiner MC, Evans R, Deacon SJ, et al. Adenine nucleotide loss in the skeletal muscles during exercise in chronic obstructive pulmonary disease. Thorax 2005; 60: 932-936.

7 Dudley GA, Terjung RL. Influence of aerobic metabolism on IMP accumulation in fast-twitch muscle. Am J Physiol 1985; 248: C37-C42.

8 Pouw EM, Schols AM, van der Vusse GJ, Wouters EF. Elevated inosine monophosphate levels in resting muscle of patients with stable chronic obstructive pulmonary disease. Am J Respir Crit Care Med 1998; 157: 453-457.

9 Buono MJ, Clancy TR, Cook JR. Blood lactate and ammonium ion accumulation during graded exercise in humans. J Appl Physiol 1984; 57: 135-139.

10 Graham TE, Bangsbo J, Gollnick PD, Juel C, Saltin B. Ammonia metabolism during intense dynamic exercise and recovery in humans. Am J Physiol 1990; 259: E170-E176.

11 Mutch BJ, Banister EW. Ammonia metabolism in exercise and fatigue: a review. Med Sci Sports Exerc 1983; 15: 41-50.

12 Rabe KF, Hurd S, Anzueto A, et al. Global strategy for the diagnosis, management, and prevention of chronic obstructive pulmonary disease: GOLD executive summary. Am J Respir Crit Care Med 2007; 176: 532-555.

13 Quanjer PH, Tammeling GJ, Cotes JE, Pedersen OF, Peslin R, Yernault JC. Lung volumes and forced ventilatory flows. Report Working Party Standardization of Lung Function Tests, European Community for Steel and Coal. Official Statement of the European Respiratory Society. Eur Respir J 1993; 6: Suppl. 16, 5-40.

14 Steiner MC, Barton RL, Singh SJ, Morgan MDL. Bedside methods versus dual energy X-ray absorptiometry for body composition measurement in COPD. Eur Respir J 2002; 19: 626-631.

15 Voorrips LE, Ravelli AC, Dongelmans PC, Deurenberg P, van Staveren WA. A physical activity questionnaire for the elderly. Med Sci Sports Exerc 1991; 23: 974-979.

16 Serres I, Gautier V, Varray A, Prefaut C. Impaired skeletal muscle endurance related to physical inactivity and altered lung function in COPD patients. Chest 1998; 113: 900-905.

17 Cooper CB, Storer TW. Exercise Testing and Interpretation. A Practical Approach. Cambridge, Cambridge University Press, 2001.

18 Greenhaff PL, Leiper JB, Ball D, Maughan RJ. The influence of dietary manipulation on plasma ammonia accumulation during incremental exercise in man. Eur J Appl Physiol 1991; 63: 338-344.

19 Lambert CP, Greenhaff PL, Ball D, Maughan RJ. Influence of sodium bicarbonate ingestion on plasma ammonia accumulation during incremental exercise in man. Eur J Appl Physiol 1993; 66: 49-54. 
20 Bergstrom J. Percutaneous needle biopsy of skeletal muscle in physiological and clinical research. Scand J Clin Lab Invest 1975; 35: 609-616.

21 Harris RC, Hultman E, Nordesjö LO. Glycogen, glycolytic intermediates and high-energy phosphates determined in biopsy samples of musculus quadriceps femoris of man at rest. Methods and variance of values. Scand J Clin Lab Invest 1974; 33: 109-120.

22 Wynants J, Van Belle H. Single-run high-performance liquid chromatography of nucleotides, nucleosides, and major purine bases and its application to different tissue extracts. Anal Biochem 1985; 144: 258-266.

23 Hultman E, Sjöholm H. Energy metabolism and contraction force of human skeletal muscle in situ during electrical stimulation. J Physiol 1983; 345: 525-532.

24 Babij P, Matthews SM, Rennie MJ. Changes in blood ammonia, lactate and amino acids in relation to workload during bicycle ergometer exercise in man. Eur J Appl Physiol Occup Physiol 1983; 50: 405-411.

25 Sahlin K, Broberg S, Ren JM. Formation of inosine monophosphate (IMP) in human skeletal muscle during incremental dynamic exercise. Acta Physiol Scand 1989; 136: 193-198.
26 Broberg S, Sahlin K. Adenine nucleotide degradation in human skeletal muscle during prolonged exercise. J Appl Physiol 1989; 67: 116-122.

27 Graham TE, MacLean DA. Ammonia and amino acid metabolism in human skeletal muscle during exercise. Can J Physiol Pharmacol 1992; 70: 132-141.

28 Dudley GA, Staron RS, Murray TF, Hagerman FC, Luginbuhl A. Muscle fiber composition and blood ammonia levels after intense exercise in humans. J Appl Physiol 1983; 54: 582-586.

29 Saey D, Michaud A, Couillard A, et al. Contractile fatigue, muscle morphometry, and blood lactate in chronic obstructive pulmonary disease. Am J Respir Crit Care Med 2005; 171: 1109-1115.

30 Harris RC, Marlin DJ, Snow DH, Harkness RA. Muscle ATP loss and lactate accumulation at different work intensities in the exercising thoroughbred horse. Eur $J$ Appl Physiol Occup Physiol 1991; 62: 235-244.

31 Yuan Y, So R, Wong S, Chan KM. Ammonia threshold comparison to lactate threshold, correlation to other physiological parameters and response to training. Scand J Med Sci Sports 2002; 12: 358-364. 